\title{
ON UNIVERSAL PRIMITIVE FUNCTIONS
}

\author{
XIAO-XIONG GAN AND KARL R. STROMBERG
}

(Communicated by Andrew Bruckner)

\begin{abstract}
This paper generalizes Marcinkiewicz's universal primitive on pointwise a.e. convergence directly to higher-dimensional spaces. It is also proved that the set of all universal primitive functions with respect to some given nonzero null sequence is residual and, hence, dense in the Banach space $C\left(I^{n}, \mathbb{R}^{m}\right) \quad \forall n, m \in \mathbb{N}$.
\end{abstract}

\section{INTRODUCTION AND DEFINITIONS}

More than fifty years ago, Marcinkiewicz [1] proved that for any nonzero null sequence $\left(h_{n}\right)_{n=1}^{\infty} \subset \mathbb{R}$ there exists a continuous function $F:[0,1] \rightarrow \mathbb{R}$ having the property: for any measurable function $\phi:[0,1] \rightarrow \mathbb{R}$, there is a subsequence $\left(h_{n_{k}}\right)_{k=1}^{\infty}$ of $\left(h_{n}\right)_{n=1}^{\infty}$ such that

$$
\lim _{k \rightarrow \infty} \frac{F\left(x+h_{n_{k}}\right)-F(x)}{h_{n_{k}}}=\phi(x) \text { a.e. on }[0,1] \text {. }
$$

This continuous function $F$ is called a universal primitive function with respect to the given sequence $\left(h_{n}\right)_{n=1}^{\infty}$.

During the past ten years, a lot of work has been done concerning analogs of Marcinkiewicz's result. In 1984, Joó [2] proved that if a bounded domain $\Omega \subset \mathbb{R}^{N}(N \geq 1)$, a nonzero null sequence $\left(h_{n}\right)_{n=1}^{\infty} \subset \mathbb{R}^{N}$, and $0 \leq p<1$ are given, then for every function $f \in L_{p}(\Omega)$, except a set of first category, the sequence

$$
\left\{\frac{f\left(x+h_{n}\right)-f(x)}{\left|h_{n}\right|}\right\}_{n=1}^{\infty}
$$

is dense in $L_{p}(\Omega)$. (Here $L_{0}(\Omega)$ is the $F$-space consisting of all measurable functions on $\Omega$ endowed with the metric of convergence in measure.)

Around three years later, Bogmér and Sövegjártó [3], Horváth [4], and Buczolich [5] independently proved that the above result fails for $p \geq 1$.

In this paper, we are going to generalize Marcinkiewicz's result on pointwise a.e. convergence directly to higher-dimensional space.

Throughout this paper, we use some definitions listed below.

Received by the editors August 13, 1992.

1991 Mathematics Subject Classification. Primary 26B35.

Key words and phrases. Universal primitive, universal function. 
(0.1) Definitions. Let $m, n \in \mathbb{N}$ and a set $\Omega$ be given.

(1) If $F: \Omega \rightarrow \mathbb{R}^{m}$ is a mapping, then for $1 \leq i \leq m$ define the $i$ th coordinate function $F_{i}: \Omega \rightarrow \mathbb{R}$ of $F$ by letting $F_{i}(t)$ be the $i$ th coordinate of $F(t)$ :

$$
F(t)=\left(F_{1}(t), F_{2}(t), \ldots, F_{m}(t)\right), \quad t \in \Omega .
$$

(2) If $\Omega$ is a topological space, then $C\left(\Omega, \mathbb{R}^{m}\right)$ denotes the family of all continuous mappings from $\Omega$ to $\mathbb{R}^{m}$. If $m=1$, write $C(\Omega)$ for $C(\Omega, \mathbb{R})$. Similarly, if $\Omega \subset \mathbb{R}^{n}$, we denote

$$
\mathfrak{M}\left(\Omega, \mathbb{R}^{m}\right)=\left\{\text { all Lebesgue measurable mappings from } \Omega \text { to } \mathbb{R}^{m}\right. \text { \} }
$$

and write $\mathfrak{M}(\Omega)$ for $\mathfrak{M}(\Omega, \mathbb{R})$.

(3) Let $\Omega \subset \mathbb{R}^{n}$, and let $f \in C(\Omega)$. If $\partial f(x) / \partial x_{j}$ exists at some $x \in \Omega^{\circ}$ (the interior of $\Omega$ ) $\forall 1 \leq j \leq n$, we define

$$
\nabla f(x)=\left(\frac{\partial}{\partial x_{1}} f(x), \frac{\partial}{\partial x_{2}} f(x), \ldots, \frac{\partial}{\partial x_{n}} f(x)\right)
$$

and call $\nabla f$ the gradient of the function $f$.

Conversely, let $f \in \mathfrak{M}\left(\Omega, \mathbb{R}^{n}\right)$. If there is $F \in C(\Omega)$ such that $\nabla F=f$, we call $F$ an antigradient of $f$.

(4) We always denote by $I^{n}$ the unit cube in $\mathbb{R}^{n}$; that is,

$$
I^{n}=\underset{k=1}{x}[0,1]=\left\{x \in \mathbb{R}^{n}: 0 \leq x_{j} \leq 1, j=1,2, \ldots, n\right\} .
$$

Also we always denote by $\lambda^{n}$ the Lebesgue outer measure on $\mathbb{R}^{n}$.

(5) Let $\Omega \subset \mathbb{R}^{n}$. The symbol $\Omega^{\circ}$ denotes the interior of $\Omega$ as a subset of $\mathbb{R}^{n}$. In particular $\left(I^{n}\right)^{\circ}=\left\{x \in \mathbb{R}^{n}: 0<x_{j}<1\right.$ for $\left.j=1, \ldots, n\right\}$. If $\phi=$ $\left(\phi_{1}, \phi_{2}, \ldots, \phi_{m}\right) \in \mathfrak{M}\left(\Omega, \mathbb{R}^{m}\right)$ and $\varepsilon>0$, then we say $\left\|\left(\phi_{1}, \phi_{2}, \ldots, \phi_{m}\right)\right\|<\varepsilon$ a.e. on $\Omega$ to mean that

$$
\lambda^{n}\left(\left\{x \in \Omega: \|\left(\phi_{1}(x), \phi_{2}(x), \ldots, \phi_{m}(x) \| \geq \varepsilon\right\}\right)=0,\right.
$$

where $\|y\|=\left(\sum_{i=1}^{m} y_{i}^{2}\right)^{1 / 2} \forall y=\left(y_{1}, y_{2}, \ldots, y_{m}\right) \in \mathbb{R}^{m}$.

(6) Let $F \in C\left(\Omega, \mathbb{R}^{m}\right)$ where $\Omega \subset \mathbb{R}^{n}$. If $\nabla F_{i}(x)$ exists at $x$ for $1 \leq i \leq m$, we denote

$$
\mathscr{J}_{F}(x)=\left(\nabla F_{1}(x), \nabla F_{2}(x), \ldots, \nabla F_{m}(x)\right)^{\mathrm{t}}
$$

that is, $\mathscr{J}_{F}(x)$ is the $m$-by- $n$ matrix having the number $D_{j} F_{i}(x)$ in its $i$ th row and $j$ th column, where $A^{t}$ denotes the transpose of the matrix $A$ and $D_{j}=\partial / \partial x_{j}$.

We call $\mathscr{J}_{F}(x)$ the Jacobian matrix of $F$ at $x$.

(7) Let $A$ and $B$ be normed linear spaces. Denote by $L(A, B)$ the set of all bounded linear transformations from $A$ to $B$. It is well known that $L(A, B)$ is also a normed linear space with the operator norm, and it is a Banach space if $B$ is a Banach space.

(8) Let $\Omega$ be an open subset of $\mathbb{R}^{n}$, let $F: \Omega \rightarrow \mathbb{R}^{m}$ be a mapping, and let $a \in \Omega$. We say that $F$ is differentiable at $a$ if there exists $T \in L\left(\mathbb{R}^{n}, \mathbb{R}^{m}\right)$ such that

$$
\lim _{\|h\| \rightarrow 0} \frac{1}{\|h\|}\|F(a+h)-F(a)-T(h)\|=0
$$

where $\|h\|=\left(\sum_{j=1}^{n} h_{j}^{2}\right)^{1 / 2} \quad \forall h=\left(h_{1}, \ldots, h_{n}\right) \in \mathbb{R}^{n}$. If such a $T$ exists, we call it the (total) differential of $F$ at $a$ and write $d F(a)=T$. 
(9) Now let us consider a mapping $\phi: \Omega \rightarrow L\left(\mathbb{R}^{n}, \mathbb{R}^{m}\right)$ for $\Omega \subset \mathbb{R}^{n}$.

We know that any $T \in L\left(\mathbb{R}^{n}, \mathbb{R}^{m}\right)$ corresponds to a real $m$-by- $n$ matrix $A_{T}=\left(a_{i j}\right)$ such that $T(x)=A_{T} x^{t}$ (matrix multiplication by a column vector) for $x=\left(x_{1}, x_{2}, \ldots, x_{n}\right) \in \Omega$; that is, the $i$ th coordinate of $T(x)$ is $\sum_{j=1}^{n} a_{i j} x_{j} \quad(i=1,2, \ldots, m)$. We shall often identify a linear transformation $T$ with its matrix $A_{T}$. Thus we may regard $L\left(\mathbb{R}^{n}, \mathbb{R}^{m}\right)$ as being the set of all $m$-by- $n$ real matrices. Hence, we can define the $i j$ th coordinate function $\phi_{i j}: \Omega \rightarrow \mathbb{R}$ of $\phi$ by letting $\phi_{i j}(x)$ be the number in the $i$ th row and $j$ th column of the matrix of $\phi(x)$.

Thus $\phi(x) \in L\left(\mathbb{R}^{n}, \mathbb{R}^{m}\right)$ can be identified with a matrix and we write

$$
\phi(x)=\left(\begin{array}{cccc}
\phi_{11}(x) & \phi_{12}(x) & \cdots & \phi_{1 n}(x) \\
\phi_{21}(x) & \phi_{22}(x) & \cdots & \phi_{2 n}(x) \\
\cdots & \cdots & \cdots & \cdots \\
\phi_{m 1}(x) & \phi_{m 2}(x) & \cdots & \phi_{m n}(x)
\end{array}\right)
$$

where each $\phi_{i j}$ is a function from $\Omega$ to $\mathbb{R}$.

(10) For any $x=\left(x_{1}, x_{2}, \ldots, x_{n}\right) \in \mathbb{R}^{n}$, we define the norms

$$
\begin{aligned}
& \|x\|_{2}=\left(\sum_{j=1}^{n} x_{j}^{2}\right)^{1 / 2}, \\
& \|x\|_{1}=\sum_{j=1}^{n}\left|x_{j}\right|, \\
& \|x\|_{\infty}=\max \left\{\left|x_{j}\right|: 1 \leq j \leq n\right\} .
\end{aligned}
$$

Let $T \in L\left(\mathbb{R}^{n}, \mathbb{R}^{m}\right)$, and let $A_{T}=\left(a_{i j}\right)_{m \times n}$ be its corresponding matrix. We define norms for $T$ by

$$
\begin{aligned}
& \|T\|_{2}=\left(\rho\left(A_{T}^{t} A_{T}\right)\right)^{1 / 2}, \\
& \|T\|_{1}=\max \left\{\sum_{i=1}^{m}\left|a_{i j}\right|: 1 \leq j \leq n\right\}, \\
& \|T\|_{\infty}=\max \left\{\sum_{j=1}^{n}\left|a_{i j}\right|: 1 \leq i \leq m\right\}
\end{aligned}
$$

where $\rho(A)$ is the spectral radius of the matrix $A$.

As usual, we say that $\phi$ is measurable (resp. continuous) if $\phi^{-1}(U)$ is Lebesgue measurable (resp. open) for each open set $U \subset L\left(\mathbb{R}^{n}, \mathbb{R}^{m}\right)$.

Thus $\phi$ is measurable if and only if each $\phi_{i j}$ is measurable and $\phi$ is continuous if and only if each $\phi_{i j}$ is continuous.

(11) For $F \in C\left(I^{n}, \mathbb{R}^{m}\right)$, define $\|F\|_{u}=\sup _{x \in I^{n}}\|F(x)\|$ where $\|y\|=$ $\left(\sum_{i=1}^{m} y_{i}^{2}\right)^{1 / 2}$ for $y \in \mathbb{R}^{m}$. This makes $C\left(I^{n}, \mathbb{R}^{m}\right)$ a Banach space. If $\left\|F_{i}\right\|_{u}<$ $\varepsilon / m$ for $i=1, \ldots, m$, then $\|F\|_{u}<\varepsilon / \sqrt{m}$.

(0.2) Remarks. (i) It is well known that all norms on $L\left(\mathbb{R}^{n}, \mathbb{R}^{m}\right)$ mentioned above are equivalent; they all generate the unique vector topology on that finitedimensional space. Therefore, the specification " $U$ open" above is unambiguous. If we do not specify the particular norm, we always mean that it is the norm $\|\cdot\|=\|\cdot\|_{2}$. 
(ii) The number $\rho(A)$ is the maximum of the moduli of the characteristic values (eigenvalues) of $A$. So

$$
\|A\|=\left(\rho\left(A^{t} A\right)\right)^{1 / 2} \leq\left(\sum_{i=1}^{m} \sum_{j=1}^{n} a_{i j}^{2}\right)^{1 / 2} .
$$

\section{SOME LEMMAS}

In order to establish the theorems in the next two section, we now present two lemmas. The first of these was proved by us as the main theorem (2.5) in [6].

(1.1) Lemma [6]. Let $n \in \mathbb{N}, f \in C\left(I^{n}, \mathbb{R}^{n}\right), F_{0} \in C\left(I^{n}\right)$, and $\varepsilon>0$ be given. Then there exists $F \in C\left(I^{n}\right)$ and an open set $W \subset\left(I^{n}\right)^{\circ}$ with $\lambda^{n}(W)=1$ such that:

(i) $\nabla F$ exists and is continuous on $W$,

(ii) $\|\nabla F(x)-f(x)\|<\varepsilon \quad \forall x \in W$, and

(iii) $\left\|F-F_{0}\right\|_{u}<\varepsilon$

where $\|y\|=\left(\sum_{j=1}^{n} y_{j}^{2}\right)^{1 / 2} \quad \forall y \in \mathbb{R}^{n}$.

(1.2) Definition. Let $n \in \mathbb{N}$. A multi-index is an $n$-tuple $\alpha=\left(\alpha_{1}, \alpha_{2}, \ldots, \alpha_{n}\right)$ of nonnegative integers. If $x=\left(x_{1}, x_{2}, \ldots, x_{n}\right) \in \mathbb{R}^{n}$ and $\alpha=\left(\alpha_{1}, \alpha_{2}, \ldots\right.$, $\left.\alpha_{n}\right)$ is a multi-index, define $x^{\alpha}=\prod_{j=1}^{n} x_{j}^{\alpha_{j}}$.

A function of the form $p(x)=\sum_{\alpha \in F} c_{\alpha} x^{\alpha} \quad\left(x \in \mathbb{R}^{n}\right)$ where $F$ is a nonvoid finite set of multi-indices and $\left\{c_{\alpha}: \alpha \in F\right\} \subset \mathbb{R}$ is called a real polynomial in $n$ real variables.

It is well known that if $\Omega \subset \mathbb{R}^{n}$ is compact, then the set of all such polynomials is dense in the real Banach space $C(\Omega)$ with its uniform norm (Weierstrass Approximation Theorem).

Let $n, m \in \mathbb{N}$. It is clear that the set $C\left(I^{n}, L\left(\mathbb{R}^{n}, \mathbb{R}^{m}\right)\right)$ of all continuous mappings $F: I^{n} \rightarrow L\left(\mathbb{R}^{n}, \mathbb{R}^{m}\right)$ is a real Banach space under the norm

$$
\|F\|=\sup _{x \in I^{n}}\|F(x)\|
$$

where the norm of the matrix $F(x)$ is as in (0.2). This is because convergence in this norm is equivalent to uniform convergence on $I^{n}$ of each component. The set $\mathscr{Q}$ of all $P=\left(p_{i j}\right)_{m \times n}$ where each component $p_{i j}$ is a polynomial as above having all its coefficients $c_{\alpha} \in \mathbb{Q}$ (and with its domain restricted to $I^{n}$ ) is a countable dense subset of $C\left(I^{n}, L\left(\mathbb{R}^{n}, \mathbb{R}^{m}\right)\right)$. We enumerate $\mathscr{Q}$ as $\left\{P_{k}\right\}_{k=1}^{\infty}$ with $P_{1}=0=(0)_{m \times n}$ for use below.

Here are some well-known facts assembled as a lemma.

(1.3) Lemma. Let $n, m \in \mathbb{N}$, let $\Omega \subset \mathbb{R}^{n}$ be open, and let $a \in \Omega$. Let $\phi: \Omega \rightarrow$ $\mathbb{R}^{m}$ be a mapping, and let $\phi_{i}$ be its ith coordinate function.

(a) Suppose that $\phi$ is differentiable at $a$. Then all of the $m n$ partial derivatives $D_{j} \phi_{i}(a)$ exist in $\mathbb{R}$, and the matrix of the linear mapping $d \phi(a)=T$ is the $m$-by- $n$ matrix $\mathscr{I}_{\phi}(a)$ having the number $D_{j} \phi_{i}(a)$ in its ith row and $j$ th column. For $h=\left(h_{1}, h_{2}, \ldots, h_{n}\right) \in \mathbb{R}^{n}$ the ith coordinate of $T(h)$ is given by

$$
T_{i}(h)=\sum_{j=1}^{n} D_{j} \phi_{i}(a) h_{j} \quad(1 \leq i \leq m) .
$$


Each $\phi_{i}$ is differentiable at a and $d \phi_{i}(a)=T_{i}$, where $T_{i}$ is defined from $\mathbb{R}^{n}$ to $\mathbb{R}$ by the above expression.

(b) If each $\phi_{i}$ is differentiable at $a$ and $d \phi_{i}(a)=T_{i}$, then $\phi$ is differentiable at $a$ and $d \phi(a)=T$, where $T$ is defined from $\mathbb{R}^{n}$ to $\mathbb{R}^{m}$ by

$$
T(h)=\left(T_{1}(h), T_{2}(h), \ldots, T_{m}(h)\right)^{t} .
$$

(c) If each of the mn partial derivatives $D_{j} \phi_{i}(1 \leq i \leq m, 1 \leq j \leq n)$ exists in $\mathbb{R}$ at every point of $\Omega$ and if the mn functions thereby defined are continuous on $\Omega$, then $\phi$ is differentiable on $\Omega$.

Proof. See (6.130) in [7].

\section{UNIVERSAL PRIMITIVE FUNCTIONS}

Now we are ready to generalize Marcinkiewicz's result on pointwise a.e. convergence directly to higher-dimensional spaces.

(2.1) Theorem. Let $n, m \in \mathbb{N}$. Let $\left\{h_{r}\right\}_{r=1}^{\infty} \subset \mathbb{R}^{n} \backslash\{0\}$ be a sequence such that $\lim _{r \rightarrow \infty}\left\|h_{r}\right\|=0$. Then there exists a mapping $F \in C\left(I^{n}, \mathbb{R}^{m}\right)$ having the following property. If $\phi: I^{n} \rightarrow L\left(\mathbb{R}^{n}, \mathbb{R}^{m}\right)$ is a measurable mapping, then there is a subsequence $\left\{s_{j}\right\}_{j=1}^{\infty}$ of $\left\{h_{r}\right\}_{r=1}^{\infty}$ such that

$$
\lim _{j \rightarrow \infty} \frac{1}{\left\|s_{j}\right\|}\left\|F\left(x+s_{j}\right)-F(x)-\phi(x)\left(s_{j}\right)\right\|=0 \text { a.e. on }\left(I^{n}\right)^{\circ}
$$

where of course $\phi(x)(h)$ is the image in $\mathbb{R}^{m}$ of the vector $h \in \mathbb{R}^{n}$ under the linear mapping $\phi(x)$.

Proof. Let $\mathscr{Q}=\left(P_{k}\right)_{k=1}^{\infty}$ be as in (1.2) above. For each $k \in \mathbb{N}$, let $P_{k, i}$ be the $i$ th row of the matrix $P_{k}$ defined in (1.2). First we show that there are five sequences:

$\left\{F_{k}\right\}_{k=0}^{\infty} \subset C\left(I^{n}, \mathbb{R}^{m}\right)$,

$\left(r_{k}\right)_{k=1}^{\infty} \subset \mathbb{N}$ with $r_{k}<r_{k+1} \quad \forall k \in \mathbb{N}$,

$\left\{t_{k}\right\}_{k=1}^{\infty}$ with $t_{k}=h_{r_{k}}$ and $\left\|t_{k+1}\right\|<\left\|t_{k}\right\| / 2 \quad \forall k \in \mathbb{N}$,

$\left\{V_{k}\right\}_{k=1}^{\infty}$ with each $V_{k}$ an open subset of $\left(I^{n}\right)^{\circ}$ and $\lambda^{n}\left(V_{k}\right)=1$, and

$\left\{E_{k}\right\}_{k=1}^{\infty}$ with $E_{k} \subset V_{k}, E_{k}$ compact, and $\lambda^{n}\left(V_{k} \backslash E_{k}\right)<2^{-k} \forall k \in \mathbb{N}$

such that

(i) $\mathscr{J}_{F_{k}}$ exists and is continuous on $V_{k}$,

(ii) $\left\|\mathscr{I}_{F_{k}}(x)-P_{k}(x)\right\|<2^{-k} \quad \forall x \in V_{k}$,

(iii) $\left\|F_{k}-F_{k-1}\right\|_{u}<\left\|t_{k-1}\right\| / k$ if $k>1$, and

(iv) $x+t_{k} \in V_{k}$ and $\frac{1}{\left\|t_{k}\right\|}\left\|F_{k}\left(x+t_{k}\right)-F_{k}(x)-d F_{k}(x)\left(t_{k}\right)\right\|<1 / k \quad \forall x \in E_{k}$, for all $k \in \mathbb{N}$.

We now inductively select the terms of these sequences.

Let $F_{0}=F_{1}=0 \in C\left(I^{n}, \mathbb{R}^{m}\right), V_{1}=\left(I^{n}\right)^{\circ}$, and $E_{1}$ be any compact subset of $V_{1}$ with $\lambda^{n}\left(E_{1}\right)>\frac{1}{2}$. Next, choose $r_{1}$ so that $x+h_{r_{1}} \in V_{1} \quad \forall x \in E_{1}$, and let $t_{1}=h_{r_{1}}$.

Suppose for some $k>1$ that $F_{k-1}, r_{k-1}, V_{k-1}$, and $E_{k-1}$ have been chosen. Write $P_{k, i}$ as the $i$ th row of the matrix $P_{k}$. Apply Lemma (1.1) to obtain $F_{k, i} \in C\left(I^{n}\right)$ and an open set $W_{i} \subset\left(I^{n}\right)^{\circ}$ with $\lambda^{n}\left(W_{i}\right)=1$ such that $\nabla F_{k, i}$ exists and is continuous on $W_{i},\left\|\nabla F_{k, i}(x)-P_{k, i}(x)\right\|<2^{-k} m^{-1}$ $\forall x \in W_{i}$, and $\left\|F_{k, i}-F_{k-1, i}\right\|_{u}<\left\|t_{k-1}\right\| / k m$ for $i=1,2, \ldots, m$. Define 
$F_{k}: I^{n} \rightarrow \mathbb{R}^{m}$ to be the mapping whose $i$ th coordinate function is $F_{k, i}$, and define $V_{k}=\bigcap_{i=1}^{m} W_{i}$. Plainly,

(1) $V_{k} \subset\left(I^{n}\right)^{\circ}, V_{k}$ is open, and $\lambda^{n}\left(V_{k}\right)=1$;

(2) $\mathscr{J}_{F_{k}}$ exists and is continuous on $V_{k}$, so $F_{k}$ is differentiable on $V_{k}$ [see $(1.3)$ ] - this is $(\mathrm{i})$;

(3) $\left\|d F_{k}(x)-P_{k}(x)\right\|<2^{-k} \forall x \in V_{k}$ [see (1.3) and (0.2) (ii)] - this is (ii); and

(4) $\left\|F_{k}-F_{k-1}\right\|_{u}<\left\|t_{k-1}\right\| / k$-this is (iii).

By (2) and the definition (8) in (0.1), $x \in V_{k} \Rightarrow$

$$
\lim _{r \rightarrow \infty} \frac{1}{\left\|h_{r}\right\|}\left\|F_{k}\left(x+h_{r}\right)-F_{k}(x)-d F_{k}(x)\left(h_{r}\right)\right\|=0 .
$$

Choose a compact set $D_{k} \subset V_{k}$ with $\lambda^{n}\left(V_{k} \backslash D_{k}\right)<2^{-k-1}$ and then find $N \in \mathbb{N}$ such that $x+h_{r} \in V_{k}$ whenever $r>N$ and $x \in D_{k}$. Now apply Egorov's Theorem (see (11.32) of [8]) to find a compact set $E_{k} \subset D_{k}$ such that

(5) $\lambda\left(V_{k} \backslash E_{k}\right)<2^{-k}$ and the limit in $(*)$ is uniform over $x \in E_{k}$. Thus there exists $r_{k}>N$ such that $r_{k}>r_{k-1}$,

(6) $\left\|t_{k}\right\|<\frac{1}{2}\left\|t_{k-1}\right\|\left(\right.$ recall $\left.t_{k}=h_{r_{k}}\right), x+t_{k} \in V_{k}$, and

(7) $\frac{1}{\left\|t_{k}\right\|}\left\|F_{k}\left(x+t_{k}\right)-F_{k}(x)-d F_{k}(x)\left(t_{k}\right)\right\|<\frac{1}{k} \quad \forall x \in E_{k}$;

this is (iv). Thus we complete our inductive selection of the sequences mentioned above and have (1)-(7) $\forall k \in \mathbb{N}$.

Since

$$
\left\|d F_{k}(x)(h)-P_{k}(x)(h)\right\| \leq\left\|d F_{k}(x)-P_{k}(x)\right\| \cdot\|h\| \stackrel{(3)}{\leq} 2^{-k}\|h\| \quad \forall h \in \mathbb{R}^{n},
$$

(7) yields

(8) $\frac{1}{\left\|t_{k}\right\|}\left\|F_{k}\left(x+t_{k}\right)-F_{k}(x)-P_{k}(x)\left(t_{k}\right)\right\|<k^{-1}+2^{-k}<\frac{2}{k} \quad \forall x \in E_{k}$.

From (4) and (6) we see that $k, l \in \mathbb{N} \Rightarrow$

$$
\begin{aligned}
\left\|F_{k+l}-F_{k}\right\|_{u} & \leq \sum_{j=k+1}^{k+l}\left\|F_{j}-F_{j-1}\right\|_{u}<\sum_{j=k+1}^{k+l} \frac{\left\|t_{j-1}\right\|}{j} \\
& <\sum_{j=k+1}^{k+l} \frac{2^{-j+k+1}\left\|t_{k}\right\|}{k+1}<2 \cdot \frac{\left\|t_{k}\right\|}{k} \leq 2 \cdot \frac{\left\|t_{1}\right\|}{k} .
\end{aligned}
$$

Thus completeness produces an $F \in C\left(I^{n}, \mathbb{R}^{m}\right)$ such that

$$
\frac{1}{\left\|t_{k}\right\|}\left\|F-F_{k}\right\|_{u} \leq \frac{2}{k} \quad \forall k \in \mathbb{N} .
$$

This inequality and $(8)$ yield

(9) $\frac{1}{\left\|t_{k}\right\|}\left\|F\left(x+t_{k}\right)-F(x)-P_{k}(x)\left(t_{k}\right)\right\|<6 / k \quad \forall x \in E_{k}, \forall k \in \mathbb{N}$.

Put $B=\bigcup_{p=1}^{\infty} \bigcap_{k=p}^{\infty} E_{k}$. Then $B \subset\left(I^{n}\right)^{\circ}$ and $I^{n} \backslash B \subset \bigcup_{k=p}^{\infty}\left(I^{n} \backslash E_{k}\right) \quad \forall p \in \mathbb{N}$ so $\lambda^{n}\left(I^{n} \backslash B\right)=0$ and $\lambda^{n}(B)=1$. From (9), it follows that $x \in B \Rightarrow \exists p(x) \in \mathbb{N}$ such that

(10) $\frac{1}{\left\|t_{k}\right\|}\left\|F\left(x+t_{k}\right)-F(x)-P_{k}(x)\left(t_{k}\right)\right\|<6 / k \quad \forall k \geq p(x)$.

Now let any measurable mapping $\phi: I^{n} \rightarrow L\left(\mathbb{R}^{n}, \mathbb{R}^{m}\right)$ be given. By applying Luzin's Theorem and the Weierstrass Approximation Theorem to each of the $m n$ component functions of the matrix-valued mapping $\phi$, we inductively find 
integers $k_{j} \in \mathbb{N}$ and compact sets $A_{j} \subset\left(I^{n}\right)^{\circ}(j=1,2, \ldots)$ such that $k_{j}<$ $k_{j+1}, \lambda^{n}\left(I^{n} \backslash A_{j}\right)<2^{-j}$, and

(11) $\left\|\phi(x)-P_{k_{j}}(x)\right\|<1 / j \quad \forall x \in A_{j}$.

Let $A=\bigcup_{q=1}^{\infty} \bigcap_{j=q}^{\infty} A_{j}$. Then $A \subset I^{n}$ and $I^{n} \backslash A \subset \bigcup_{j=q}^{\infty}\left(I^{n} \backslash A_{j}\right) \quad \forall q \in \mathbb{N}$, so $\lambda^{n}\left(I^{n} \backslash A\right)=0$ and $\lambda^{n}(A)=1$.

If $x \in A$, then (11) yields $q(x) \in \mathbb{N}$ such that

(12) $\frac{1}{\left\|t_{k_{j}}\right\|}\left\|\phi(x)\left(t_{k_{j}}\right)-P_{k_{j}}(x)\left(t_{k_{j}}\right)\right\| \leq\left\|\phi(x)-P_{k_{j}}(x)\right\|<1 / j \quad \forall j \geq q(x)$.

Write $s_{j}=t_{k_{j}}$. Then $\left\{s_{j}\right\}_{j=1}^{\infty}$ is a subsequence of $\left\{h_{r}\right\}_{r=1}^{\infty}$ and (10) and (12) show that if $x \in A \cap B$ and $j \geq \max \{p(x), q(x)\}$ (hence $k_{j} \geq p(x)$ ), then

$$
\frac{1}{\left\|s_{j}\right\|}\left\|F\left(x+s_{j}\right)-F(x)-\phi(x)\left(s_{j}\right)\right\|<\frac{6}{j}+\frac{1}{j}=\frac{7}{j} \text {. }
$$

Since $\lambda^{n}(A \cap B)=1$ and $\phi$ was arbitrary, the proof is complete.

(2.2) Remark. It is clear that (2.1) remains true if we replace all three occurrences of $I^{n}$ in its statement with any rectangular box $B=\times_{j=1}^{n}\left[a_{j}, b_{j}\right]$ where $a_{j}<b_{j}$ in $\mathbb{R}$ for $j=1,2, \ldots, n$. It suffices to consider composites with the nonsingular affine transformation $T: \mathbb{R}^{n} \rightarrow \mathbb{R}^{n}$ given by $T(x)=y$ where $y_{j}=\left(x_{j}-a_{j}\right) /\left(b_{j}-a_{j}\right)$ which maps $B$ onto $I^{n}$.

\section{DENSITY IN $C\left(I^{n}, \mathbb{R}^{m}\right)$ OF UNIVERSAL PRIMITIVE FUNCTIONS}

Finally, we examine the set of all $F$ that correspond to a given sequence $\left\{h_{r}\right\}_{r=1}^{\infty} \subset \mathbb{R}^{n}$ as in (2.1).

(3.1) Definition. Let $m, n \in \mathbb{N}$, and let $\left\{h_{r}\right\}_{r=1}^{\infty}$ be as in (2.1): $0 \neq h_{r} \in \mathbb{R}^{n}$ $\forall r \in \mathbb{N}$ and $\lim _{r \rightarrow \infty}\left\|h_{r}\right\|=0$. Each $F \in C\left(I^{n}, \mathbb{R}^{m}\right)$ having the property cited in (2.1) is called a universal primitive function relative to $\left\{h_{r}\right\}$, and we denote the set of all such functions $F$ by $U\left(\left\{h_{r}\right\}\right)$. We use $U^{\prime}\left(\left\{h_{r}\right\}\right)$ to denote the set of all $f \in C\left(I^{n}, \mathbb{R}^{m}\right)$ having the property: whenever $k, l \in \mathbb{N}$ there exists some $r>k$ such that

$$
\lambda^{n}\left(\left\{x \in I^{n}: x+h_{r} \in I^{n}, \frac{1}{\left\|h_{r}\right\|}\left\|f\left(x+h_{r}\right)-f(x)-P_{l}(x)\left(h_{r}\right)\right\| \geq \frac{1}{k}\right\}\right)<\frac{1}{k}
$$

where $P_{l}$ is as in (1.3).

(3.2) Lemma. Let $m, n \in \mathbb{N}$, and let $\left\{h_{r}\right\}_{r=1}^{\infty} \subset \mathbb{R}^{n} \backslash\{0\}$ be a null sequence. Then $U\left(\left\{h_{r}\right\}\right)=U^{\prime}\left(\left\{h_{r}\right\}\right)$.

Proof. Suppose $F \in U\left(\left\{h_{r}\right\}\right)$ is given. Then for each $l \in \mathbb{N}$, there exists a subsequence $\left\{h_{r_{j}}\right\}_{j=1}^{\infty}$ of $\left\{h_{r}\right\}_{r=1}^{\infty}$, which only depends on $P_{l}$ and $F$, such that

$$
\lim _{j \rightarrow \infty} \frac{1}{\left\|h_{r_{j}}\right\|}\left\|F\left(x+h_{r_{j}}\right)-F(x)-P_{l}(x)\left(h_{r_{j}}\right)\right\|=0 \text { a.e. on }\left(I^{n}\right)^{\circ} \text {. }
$$

Let $k, l \in \mathbb{N}$ be given; then Egorov's Theorem yields a compact set $E_{l} \subset$ $\left(I^{n}\right)^{\circ}$ with $\lambda\left(E_{l}\right)>1-\frac{1}{k}$ and the limit in $(*)$ is uniform on $E_{l}$. Thus there exists $r>k$ such that $x+h_{r} \in I^{n}$ and

$$
\frac{1}{\left\|h_{r}\right\|}\left\|F\left(x+h_{r}\right)-F(x)-P_{l}(x)\left(h_{r}\right)\right\|<\frac{1}{k}
$$

for all $x \in I^{n} \backslash E_{l}$. Thus $F \in U^{\prime}\left(\left\{h_{r}\right\}\right)$. 
Now suppose $F \in U^{\prime}\left(\left\{h_{r}\right\}\right)$ is given.

Let any measurable mapping $\phi: I^{n} \rightarrow L\left(\mathbb{R}^{n}, \mathbb{R}^{m}\right)$ be given. Use exactly the same argument as for the proof of (11) in (2.1): By applying Luzin's Theorem and the Weierstrass Approximation Theorem to each of the $m n$ component functions of the matrix-valued mapping $\phi$, we get integers $k_{j} \in \mathbb{N}$ and compact sets $A_{j} \subset\left(I^{n}\right)^{\circ}(j=1,2, \ldots)$ such that $k_{j}<k_{j+1}, \lambda^{n}\left(I^{n} \backslash A_{j}\right)<2^{-j}$, and

$$
\left\|\phi(x)-P_{k_{j}}(x)\right\|<1 / j \quad \forall x \in A_{j} .
$$

Since $F \in U^{\prime}\left(\left\{h_{r}\right\}\right)$, we can inductively find integers $r_{j} \in \mathbb{N}$ and measurable sets $B_{j} \subset\left(I^{n}\right)^{\circ}(j=1,2, \ldots)$ such that $r_{j}<r_{j+1}, \lambda^{n}\left(I^{n} \backslash B_{j}\right)<2^{-j}, x+h_{r_{j}} \in$ $I^{n}$, and

$$
(* * *) \quad \frac{1}{\left\|h_{r_{j}}\right\|}\left\|F\left(x+h_{r_{j}}\right)-F(x)-P_{k_{j}}(x)\left(h_{r_{j}}\right)\right\|<2^{-j} \quad \forall x \in B_{j} .
$$

Let $C_{j}=A_{j} \cap B_{j}$ for all $j \in \mathbb{N}$. Then $\lambda^{n}\left(I^{n} \backslash C_{j}\right)<2^{-j+1}$. If $x \in C_{j}$, then $(* *)$ and $(* * *)$ imply

$$
\begin{aligned}
\frac{1}{\left\|h_{r_{j}}\right\|}\left\|F\left(x+h_{r_{j}}\right)-F(x)-\phi(x)\left(h_{r_{j}}\right)\right\| \\
\quad \leq \frac{1}{\left\|h_{r_{j}}\right\|}\left(\left\|F\left(x+h_{r_{j}}\right)-F(x)-P_{k_{j}}(x)\left(h_{r_{j}}\right)\right\|+\left\|\left(\phi(x)-P_{k_{j}}(x)\right)\left(h_{r_{j}}\right)\right\|\right) \\
\quad<2^{-j}+\frac{1}{\left\|h_{r_{j}}\right\|}\left\|\phi(x)-P_{k_{j}}(x)\right\| \cdot\left\|h_{r_{j}}\right\| \leq \frac{2}{j} .
\end{aligned}
$$

Take $C=\bigcup_{l=1}^{\infty} \bigcap_{j=l}^{\infty} C_{j}$. Then $C \subset\left(I^{n}\right)^{\circ}$ and $I^{n} \backslash C \subset \bigcup_{j=l}^{\infty}\left(I^{n} \backslash C_{j}\right) \quad \forall l \in \mathbb{N}$, so $\lambda^{n}\left(I^{n} \backslash C\right)=0$ and $\lambda^{n}(C)=1$.

If $x \in C$, then there is some $j_{0} \in \mathbb{N}$ such that $x \in C_{j} \forall j \geq j_{0}$. Thus

$$
\frac{1}{\left\|h_{r_{j}}\right\|}\left\|F\left(x+h_{r_{j}}\right)-F(x)-\phi(x)\left(h_{r_{j}}\right)\right\|<\frac{2}{j} \quad \forall j \geq j_{0} .
$$

Consequently

$$
\lim _{j \rightarrow \infty} \frac{1}{\left\|h_{r_{j}}\right\|}\left\|F\left(x+h_{r_{j}}\right)-F(x)-\phi(x)\left(h_{r_{j}}\right)\right\|=0 \quad \text { a.e. on }\left(I^{n}\right)^{\circ} \text {. }
$$

This proves that $F \in U\left(\left\{h_{r}\right\}\right)$ and completes the proof.

(3.3) Theorem. Let $m, n \in \mathbb{N}$, and let $\left\{h_{r}\right\}_{r=1}^{\infty} \subset \mathbb{R}^{n} \backslash\{0\}$ be a null sequence. Then $U\left(\left\{\left(h_{r}\right)\right\}\right)$ is residual (in the sense of Baire category) and hence dense in the Banach space $C\left(I^{n}, \mathbb{R}^{m}\right)$.

Proof. Let $F \in C\left(I^{n}, \mathbb{R}^{m}\right) \backslash U\left(\left\{h_{r}\right\}\right)$. Then $F \notin U^{\prime}\left(\left\{h_{r}\right\}\right)$ by (3.2). Thus there are some $s, t \in \mathbb{N}$ such that, for all $p>t$, we have

(1) $\lambda^{n}\left(\left\{x \in I^{n}: x+h_{p} \in I^{n}, \frac{1}{\left\|h_{p}\right\|}\left\|F\left(x+h_{p}\right)-F(x)-P_{s}(x)\left(h_{p}\right)\right\| \geq \frac{1}{t}\right\}\right) \geq \frac{1}{t}$, where $P_{s}$ is as in (1.2).

Define $\mathscr{A}_{s, t}$ to be the set of all $F \in C\left(I^{n}, \mathbb{R}^{m}\right)$ such that (1) holds $\forall p>t$, and let $\mathscr{A}=\bigcup_{s, t \in \mathbb{N}} \mathscr{A}_{s, t}$. We have just seen that $\mathscr{A} \supset C\left(I^{n}, \mathbb{R}^{m}\right) \backslash U\left(\left\{h_{r}\right\}\right)$. It is obvious that $\mathscr{A} \subset C\left(I^{n}, \mathbb{R}^{m}\right) \backslash U\left(\left\{h_{r}\right\}\right)$ and hence $\mathscr{A}=C\left(I^{n}, \mathbb{R}^{m}\right) \backslash U\left(\left\{h_{r}\right\}\right)$. Thus, it suffices to show that each $\mathscr{A}_{s, t}$ is nowhere dense in $C\left(I^{n}, \mathbb{R}^{m}\right)$ with respect to the norm $\|\cdot\|_{u}$. We first show that each $\mathscr{A}_{s, t}$ is closed. 
Let $s, t \in \mathbb{N}$ be given, and let $\left(F_{j}\right)_{j=1}^{\infty} \subset \mathscr{A}_{s, t}$ with $\lim _{j \rightarrow \infty}\left\|F_{j}-F\right\|_{u}=0$ for some $F \in C\left(I^{n}, \mathbb{R}^{m}\right)$. For each $p \in \mathbb{N}$ define

$$
D_{p}=\left\{x \in I^{n}: x+h_{p} \in I^{n}, \frac{1}{\left\|h_{p}\right\|}\left\|F\left(x+h_{p}\right)-F(x)-P_{s}(x)\left(h_{p}\right)\right\| \geq \frac{1}{t}\right\} .
$$

Assume $F \notin \mathscr{A}_{s, t}$. Then there exists some $p>t$ such that $\lambda^{n}\left(D_{p}\right)<\frac{1}{t}$. Of course we can find some $\varepsilon>0$ so that $\lambda^{n}\left(D_{p}\right)<\frac{1}{t}-\varepsilon$.

Choose a relatively open subset $V$ of $\left(I^{n}\right)^{\circ}$ such that

$$
D_{p} \subset V \text { and } \lambda^{n}(V)<\frac{1}{t}-\frac{\varepsilon}{2}
$$

Let $W=I^{n} \backslash V$. Then $W$ is compact and

$$
\frac{1}{\left\|h_{p}\right\|}\left\|F\left(x+h_{p}\right)-F(x)-P_{s}(x)\left(h_{p}\right)\right\|<\frac{1}{t} \quad \text { if } x \in W \text { and } x+h_{p} \in I^{n} .
$$

By compactness and continuity there exists some $k \in \mathbb{N}$, such that

$$
\frac{1}{\left\|h_{p}\right\|}\left\|F\left(x+h_{p}\right)-F(x)-P_{s}(x)\left(h_{p}\right)\right\|<\frac{1}{t}-\frac{\varepsilon}{k} \quad \text { if } x \in W \text { and } x+h_{p} \in I^{n} .
$$

Now we choose $j \in \mathbb{N}$ so that

$$
\left\|F_{j}-F\right\|<\frac{1}{4 k} \varepsilon \cdot\left\|h_{p}\right\| .
$$

Then $x \in I^{n} \backslash V=W$ and $x+h_{p} \in I^{n} \Rightarrow$

$$
\begin{aligned}
\frac{1}{\left\|h_{p}\right\|} \| & F_{j}\left(x+h_{p}\right)-F_{j}(x)-P_{s}(x)\left(h_{p}\right) \| \\
\leq & \frac{1}{\left\|h_{p}\right\|}\left\|F\left(x+h_{p}\right)-F(x)-P_{s}(x)\left(h_{p}\right)\right\| \\
& +\frac{1}{\left\|h_{p}\right\|}\left\|F_{j}\left(x+h_{p}\right)-F\left(x+h_{p}\right)\right\|+\frac{1}{\left\|h_{p}\right\|}\left\|F_{j}(x)-F(x)\right\| \\
\leq & \frac{1}{\left\|h_{p}\right\|}\left\|F\left(x+h_{p}\right)-F(x)-P_{s}(x)\left(h_{p}\right)\right\|+\frac{\varepsilon}{4 k}+\frac{\varepsilon}{4 k} \\
< & \frac{1}{t}-\frac{\varepsilon}{k}+\frac{\varepsilon}{2 k}<\frac{1}{t} .
\end{aligned}
$$

Since $\lambda^{n}(V)<\frac{1}{t}-\frac{\varepsilon}{2}$, it follows that, with $F_{j}$ in place of $F$, the set on the left side of (1) lies in $V$, so its measure is less than $\frac{1}{t}$. Thus $F_{j} \notin \mathscr{A}_{s, t}$.

This contradiction proves that $F \in \mathscr{A}_{s, t}$, and hence $\mathscr{A}_{s, t}$ is closed.

Next we show that $\left(\mathscr{A}_{s, t}\right)^{\circ}=\varnothing$.

Fix any $F \in \mathscr{A}_{s, t}$ and any $\varepsilon$ with $0<\varepsilon<\frac{1}{t}$. We want to find $G \in$ $C\left(I^{n}, \mathbb{R}^{m}\right) \backslash \mathscr{A}_{s, t}$ such that $\|F-G\|_{u}<\varepsilon$. This will prove that $\mathscr{A}_{s, t}$ has no interior points. As before, we write

$$
F=\left(F_{1}, F_{2}, \ldots, F_{m}\right) \text { and } P_{s}=\left(P_{s, 1}, P_{s, 2}, \ldots, P_{s, m}\right)^{t}
$$

where $P_{s, i}$ is the $i$ th row of the $m \times n$ matrix $P_{s}$ defined in (1.2). Apply Lemma (1.1) to obtain an open set $W_{i} \subset\left(I^{n}\right)^{\circ}$ with $\lambda^{n}\left(W_{i}\right)=1$ and a function $G_{i} \in C\left(I^{n}\right)$ such that:

(i) $\nabla G_{i}$ exists and is continuous on $W_{i}$,

(ii) $\left\|\nabla G_{i}(x)-P_{s, i}(x)\right\|<\varepsilon / m \quad \forall x \in W_{i}$, and

(iii) $\left\|G_{i}-F_{i}\right\|_{u}<\varepsilon / m$. 
Put $W=\bigcap_{i=1}^{m} W_{i}$ and $G=\left(G_{1}, G_{2}, \ldots, G_{m}\right)$. Then $G \in C\left(I^{n}, \mathbb{R}^{m}\right)$,

(2) $W$ is open, $W \subset\left(I^{n}\right)^{\circ}, \lambda^{n}(W)=1$;

(3) $\mathscr{I}_{G}$ is continuous on $W$;

(4) $\left\|\mathscr{I}_{G}(x)-P_{s}(x)\right\|<\varepsilon \quad \forall x \in W$; and

(5) $\|G-F\|_{u}<\varepsilon$.

Now we show that $G \notin \mathscr{A}_{s, t}$.

For any $x \in W$ and $r \in \mathbb{N}$ with $x+h_{r} \in I^{n}$, (4) yields

$$
\begin{aligned}
& \frac{1}{\left\|h_{r}\right\|}\left\|G\left(x+h_{r}\right)-G(x)-P_{s}(x)\left(h_{r}\right)\right\| \\
& \quad \leq \frac{1}{\left\|h_{r}\right\|}\left\|G\left(x+h_{r}\right)-G(x)-\mathscr{I}_{G}(x)\left(h_{r}\right)\right\|+\frac{1}{\left\|h_{r}\right\|}\left\|\left(P_{s}(x)-\mathscr{I}_{G}(x)\right)\left(h_{r}\right)\right\| \\
& \quad \leq \frac{1}{\left\|h_{r}\right\|}\left\|G\left(x+h_{r}\right)-G(x)-\mathscr{I}_{G}(x)\left(h_{r}\right)\right\|+\varepsilon .
\end{aligned}
$$

By (1.3) and (3), $G$ is differentiable at every $x \in W$; hence,

$$
\lim _{r \rightarrow \infty} \frac{1}{\left\|h_{r}\right\|}\left\|G\left(x+h_{r}\right)-G(x)-\mathscr{I}_{G}(x)\left(h_{r}\right)\right\|=0 \quad \forall x \in W .
$$

We can apply Egorov's Theorem as before to obtain a compact set $E \subset W$ with $\lambda^{n}(W \backslash E)<\frac{1}{2 t}$ and some $r>t$ such that $x+h_{r} \in W$ and

$$
\frac{1}{\left\|h_{r}\right\|}\left\|G\left(x+h_{r}\right)-G(x)-\mathscr{I}_{G}(x)\left(h_{r}\right)\right\|<\frac{1}{t}-\varepsilon \quad \forall x \in E .
$$

It follows that

$$
\begin{aligned}
& \left\{x \in I^{n}: x+h_{r} \in I^{n}, \frac{1}{\left\|h_{r}\right\|}\left\|G\left(x+h_{r}\right)-G(x)-P_{s}(x)\left(h_{r}\right)\right\| \geq \frac{1}{t}\right\} \\
& \quad \subset(W \backslash E) \cup\left(I^{n} \backslash W\right)
\end{aligned}
$$

which has measure less than $\frac{1}{t}$. Comparing with (1) we see that $G \notin \mathscr{A}_{s, t}$, as we wanted to show. We conclude that $\left(\mathscr{A}_{s, t}\right)^{\circ}=\varnothing$ and so $\mathscr{A}_{s, t}$, being closed, is nowhere dense in $C\left(I^{n}, \mathbb{R}^{m}\right)$.

We have proven that $\mathscr{A}$ is of first category, and hence $U\left(\left\{h_{r}\right\}\right)$ is residual in $C\left(I^{n}, \mathbb{R}^{m}\right)$. By the Baire Category Theorem, $U\left(\left\{h_{r}\right\}\right)$ is dense in $C\left(I^{n}, \mathbb{R}^{m}\right)$.

\section{REFERENCES}

1. J. Marcinkiewicz, Sur les nombres derives, Fund. Math. 24 (1935), 305-308.

2. I. Joó, On the divergence of eigenfunction expansions, Ann. Univ. Sci. Sect. Math. 32 (1989), 2-36.

3. A. Bogmér and A. Sövegjártó, On universal functions, Acta Math. Hungar. 49 (1987), 237-239.

4. M. Horváth, On multidimensional universal functions, Studia Sci. Math. Hungar. 22 (1987), 75-78.

5. Z. Buczolich, On universal functions and series, Acta Math. Hungar. 49 (1987), 403-414.

6. Xiao-Xiong Gan and Karl R. Stromberg, On approximate antigradients, Proc. Amer. Math. Soc. (to appear). 
7. Karl R. Stromberg, An introduction to classical real analysis, Wadsworth, Belmont, CA, 1981.

8. Edwin Hewitt and Karl Stromberg, Real and abstract analysis, Springer-Verlag, New York, 1965.

Department of Mathematics, Kansas State Univerity, Manhattan, Kansas 66506

Current address, X.-X. Gan: Department of Mathematics, Morgan State University, Baltimore, Maryland 21239

E-mail address, X.-X. Gan: gan@morganvx (Bitnet) or gan@moe.morgan.edu (Internet) 